Supplement of Foss. Rec., 24, 151-169, 2021

https://doi.org/10.5194/fr-24-151-2021-supplement

(C) Author(s) 2021. CC BY 4.0 License.

Supplement of

\title{
Determining the gait of Miocene, Pliocene, and Pleistocene horses from fossilized trackways
}

\author{
Alan Vincelette \\ Correspondence to: Alan Vincelette (avincelette@stjohnsem.edu) \\ The copyright of individual parts of the supplement might differ from the article licence.
}


Table S1: Basic information about the modern horses (Equus caballus) studied. The V superscript attached to the horse number here and below indicates video footage was taken and the K superscript here and below indicates that the measurements were extrapolated from material in Kienapfel, et al., 2014, fig. 4a (slow walk), 4b (fast walk), 5a (slow tölt), 5b (fast tölt), 6a (interpreted as fast trot), 6b (interpreted as slow trot), 7a (pace), 8a (canter), 8b (canter), and 8c (gallop).

\begin{tabular}{|llll|}
\hline Horse & Breed & $\begin{array}{l}\text { Height at } \\
\text { withers } \\
(\mathrm{m})\end{array}$ & $\begin{array}{l}\text { Hoof } \\
\text { length } \\
\text { (cm) }\end{array}$ \\
\hline 1 & Arabian & 1.63 & 12.7 \\
2 & Andalusian/Quarter & 1.54 & 12.7 \\
3 & Thoroughbred & 1.65 & 12.7 \\
$4^{\mathrm{V}}$ & Arabian & 1.45 & 12.7 \\
5 & Arabian & 1.53 & 15.2 \\
6 & Arabian & 1.53 & $14.0 \quad 15$ \\
$7^{\mathrm{V}}$ & Quarter & 1.50 & 12.7 \\
8 & Thoroughbred & 1.63 & 16.5 \\
9 & Andalusian/Quarter & 1.63 & 14.0 \\
$10^{\mathrm{V}}$ & Arabian/Quarter & 1.58 & 12.7 \\
$11^{\mathrm{V}}$ & Quarter & 1.63 & 14.0 \\
12 & Tennessee Walker & 1.53 & ca. 1520 \\
13 & Tennessee Walker & 1.63 & 14.0 \\
14 & Quarter & 1.54 & 15.2 \\
$15^{\mathrm{V}}$ & American Saddlebred & 1.55 & 16.5 \\
& & & \\
$16^{\mathrm{V}}$ & Tennessee Walker & 1.54 & ca. 14.0 \\
$17^{\mathrm{V}}$ & Tennessee Walker & ca. 1.68 & ca. 1425 \\
$18^{\mathrm{V}}$ & Rocky Mountain & ca. 1.47 & ca. 12.7 \\
$19^{\mathrm{V}}$ & Tennessee Walker & 1.63 & ca. 15.2 \\
$20^{\mathrm{V}}$ & Icelandic & ca. 1.40 & ca. 10.0 \\
& & & \\
$21^{\mathrm{K}}$ & German Warmblood & ca. 1.68 & ca. 12.7 \\
$22^{\mathrm{K}}$ & Icelandic & 1.37 & ca. 1030 \\
$23^{\mathrm{K}}$ & Icelandic & ca. 1.38 & ca. 10.0 \\
$24^{\mathrm{K}}$ & Paso Fino & 1.65 & ca. 12.7 \\
$25^{\mathrm{K}}$ & German Saddle & 1.50 & ca. 12.7 \\
\hline & & & \\
\hline
\end{tabular}


Tables S2-S10: Measurements of gaits in modern horse footprints (Equus caballus). Speed is calculated from formula found in Alexander (1976): Estimated speed $(\mathrm{m} / \mathrm{s})=1.565(\mathrm{~m} / \mathrm{s} 2) \mathrm{x}$ stride (cycle) length (m)1.67 x height (m)-1.17. Positive values for overtrack column indicate overtracking (overstepping). Negative values for overtrack are quantitative and indicate capping (overlapping) of hooves and eventual undertracking (understepping) once the length of hoof is exceeded. Negative values for lateral offset, on the other hand, are vector signs and indicate that the couplets point in an inward direction while positive values indicate that the couplets point in an outward direction. Hence absolute values of the lateral offset are used to calculate average values.

Table S2: Measurements of the slow walk.

\begin{tabular}{|lllllll|} 
Horse & $\begin{array}{l}\text { Calculated } \\
\text { speed }(\mathrm{m} / \mathrm{s})\end{array}$ & $\begin{array}{l}\text { Cycle } \\
\text { length }(\mathrm{cm})\end{array}$ & $\begin{array}{l}\text { Intercouplet } \\
\text { distance }(\mathrm{cm})\end{array}$ & $\begin{array}{l}\text { Overtrack } \\
(\mathrm{cm})\end{array}$ & $\begin{array}{l}\text { Avg. interior } \\
\text { straddle }(\mathrm{cm})\end{array}$ & $\begin{array}{l}\text { L/R lateral offset } \\
\text { (Cross measure })(\mathrm{cm})\end{array}$ \\
\hline 1 & 1.32 & 127.0 & $49.5,52.1$ & $-12.7,-12.7$ & 7.6 & $4.1(4.1), 0(0)$ \\
2 & 1.55 & 134.6 & $48.3,48.3$ & $-11.4,-11.4$ & 0 & $5.1(5.3), 0(1.3)$ \\
3 & 1.76 & 152.4 & $61.0,61.0$ & $-10.2,-10.2$ & 0 & $0(10.9), 3.8(10.9)$ \\
4 & 1.79 & 141.0 & $36.8,40.6$ & $-19.1,-19.1$ & 2.5 & $0(19.1), 5.1(19.7)$ \\
5 & 1.92 & 152.4 & $61.0,61.0$ & $-15.2,-15.2$ & 3.8 & $0(0), 6.1(6.1)$ \\
6 & 2.08 & 160.0 & $53.3,53.3$ & $-2.5,-2.5$ & 6.4 & $0(13.0), 0(13.0)$ \\
7 & 2.19 & 162.6 & $55.9,55.9$ & 0,0 & 5.1 & $0(12.7), 0(12.7)$ \\
$21^{\mathrm{K}}$ & ca. 1.61 & ca. 146.3 & ca. $60.0,37.5$ & ca. 0,0 & ca. -5.1 & ca. $2.5(13.2), 0(12.7)$ \\
\hline
\end{tabular}

Table S3: Measurements of the fast walk

\begin{tabular}{|lll|llll|} 
Horse & $\begin{array}{l}\text { Calculated } \\
\text { speed }(\mathrm{m} / \mathrm{s})\end{array}$ & $\begin{array}{l}\text { Cycle } \\
\text { length }(\mathrm{cm})\end{array}$ & $\begin{array}{l}\text { Intercouplet } \\
\text { distance }(\mathrm{cm})\end{array}$ & $\begin{array}{l}\text { Overtrack } \\
(\mathrm{cm})\end{array}$ & $\begin{array}{l}\text { Avg. interior } \\
\text { straddle }(\mathrm{cm})\end{array}$ & $\begin{array}{l}\text { L/R lateral offset } \\
\text { (Cross measure) }(\mathrm{cm})\end{array}$ \\
\hline 8 & 2.20 & 172.4 & $55.9,63.5$ & $-7.6,0$ & 0.0 & $7.6(12.7), 0(16.5)$ \\
9 & 2.29 & 176.7 & $55.9,55.9$ & $2.5,6.4$ & 0 & $-6.4(17.7), 6.4(21.2)$ \\
3 & 2.29 & 178.0 & $57.2,57.2$ & $6.4,6.4$ & 6.4 & $0(19.1),-5.1(19.9)$ \\
10 & 2.32 & 173.8 & $47.0,57.2$ & $3.8,5.1$ & 2.5 & $3.2(16.7), 1.3(17.8)$ \\
11 & 2.37 & 180.4 & $58.4,58.4$ & $2.5,5.1$ & ca. 0.0 & $0(16.5)$, c. $1.3(19.1)$ \\
6 & 2.53 & 180.4 & $59.7,59.7$ & $2.5,2.5$ & -0.6 & $0(16.5),-5.7(17.4)$ \\
& & & & & & \\
$21^{\mathrm{K}}$ & ca. 2.72 & ca. 200.3 & ca. $52.5,67.5$ & ca. $15,7.5$ & ca. -5.1 & ca. $-2.5(15.2), 2.5(8.1)$ \\
\hline
\end{tabular}

Table S4: Measurements of the running walk

\begin{tabular}{|llllll|} 
Horse & $\begin{array}{l}\text { Calculated } \\
\text { speed }(\mathrm{m} / \mathrm{s})\end{array}$ & $\begin{array}{l}\text { Cycle } \\
\text { Length }(\mathrm{cm})\end{array}$ & $\begin{array}{l}\text { Intercouplet } \\
\text { distance }(\mathrm{cm})\end{array}$ & $\begin{array}{l}\text { Overtrack } \\
(\mathrm{cm})\end{array}$ & $\begin{array}{l}\text { Avg. interior } \\
\text { straddle }(\mathrm{cm})\end{array}$ \\
\hline 12 & 3.23 & ca. 208.1 & $35.6,48.3$ & $24.1,24.1$ & ca. 0.0 \\
13 & 3.24 & 217.2 & $43.2,43.2$ & $25.4,25.4$ & 2.5 \\
\hline
\end{tabular}


Table S5: Measurements of the slow trot

\begin{tabular}{|c|c|c|c|c|c|c|}
\hline Horse & $\begin{array}{l}\text { Calculated } \\
\text { speed }(\mathrm{m} / \mathrm{s})\end{array}$ & $\begin{array}{l}\text { Cycle } \\
\text { length }(\mathrm{cm})\end{array}$ & $\begin{array}{l}\text { Intercouplet } \\
\text { distance }(\mathrm{cm})\end{array}$ & $\begin{array}{l}\text { Overtrack } \\
(\mathrm{cm})\end{array}$ & $\begin{array}{l}\text { Avg. interior } \\
\text { straddle }(\mathrm{cm})\end{array}$ & $\begin{array}{l}\text { L/R Lateral offset } \\
\text { (Cross measure) }(\mathrm{cm})\end{array}$ \\
\hline 9 & 2.17 & 170.8 & $63.5,63.5$ & $-19.1,-21.6$ & 6.4 & $7.6(20.5), 0(21.6)$ \\
\hline 8 & 2.54 & 187.9 & $58.4,63.5$ & 0,0 & 0 & $2.5(16.7), 0(16.5)$ \\
\hline 2 & 2.62 & 184.2 & $78.7,78.7$ & $-11.4,-11.4$ & 6.4 & $0(1.3), 3.8(4.0)$ \\
\hline 1 & 2.67 & 193.7 & $74.3,76.2$ & $-3.8,-3.8$ & 2.5 & $7.6(11.7), 0(8.9)$ \\
\hline 6 & 3.30 & 210.8 & $71.1,71.1$ & $-20.3,-20.3$ & 1.3 & $-8.9(22.0), 0(20.3)$ \\
\hline 7 & 3.52 & 216.0 & $76.2,76.2$ & $-19.1,-19.1$ & 0 & $0(19.1), 5.1(19.8)$ \\
\hline $21^{\mathrm{K}}$ & ca. 3.58 & ca. 236.0 & ca. $93.8,93.8$ & ca. $-6.4,-2.5$ & ca. -0.01 & ca. $-6.4(9.0), 0(10.2)$ \\
\hline
\end{tabular}

Table S6: Measurements of the fast trot

\begin{tabular}{|lllllll|} 
Horse & $\begin{array}{l}\text { Calculated } \\
\text { speed }(\mathrm{m} / \mathrm{s})\end{array}$ & $\begin{array}{l}\text { Cycle } \\
\text { length }(\mathrm{cm})\end{array}$ & $\begin{array}{l}\text { Intercouplet } \\
\text { Distance }(\mathrm{cm})\end{array}$ & $\begin{array}{l}\text { Overtrack } \\
(\mathrm{cm})\end{array}$ & $\begin{array}{l}\text { Avg. interior } \\
\text { straddle }(\mathrm{cm})\end{array}$ & $\begin{array}{l}\text { L/R Lateral offset } \\
\text { (Cross measure) }(\mathrm{cm})\end{array}$ \\
\hline 14 & 3.81 & 231.0 & $88.9,88.9$ & $-3.8,-3.8$ & 2.0 & $-10.2(15.3), 12.7(17.1)$ \\
3 & 4.86 & 279.4 & $106.7,106.7$ & $-7.6,-7.6$ & 2.5 & $-7.6(9.1), 0(5.1)$ \\
4 & 5.03 & 261.5 & $97.8,104.1$ & $3.8,5.1$ & ca. 0 & ca. $2.5(17.3), 0(17.1)$ \\
6 & 5.11 & 274.4 & $104.1,104.1$ & $5.1,5.1$ & 0.6 & $-6.4(15.2), 10.2(17.2)$ \\
10 & 5.12 & 279.4 & $111.8,116.8$ & 0,0 & 6.4 & $0(12.7), 0(12.7)$ \\
& & & & & & \\
$21^{\mathrm{K}}$ & ca. 5.12 & ca. 292.5 & ca. $116.3,105.0$ & ca. $9.4,8.0$ & ca. -2.0 & ca. $0(22.1),-2(20.8)$ \\
\hline
\end{tabular}

Table S7: Measurements of the slow gait (15a) and rack/tölt (15b, 16, 17, 20, and 22a and 22b)

\begin{tabular}{|lllllll|} 
Horse & $\begin{array}{l}\text { Calculated } \\
\text { speed }(\mathrm{m} / \mathrm{s})\end{array}$ & $\begin{array}{l}\text { Cycle } \\
\text { length }(\mathrm{cm})\end{array}$ & $\begin{array}{l}\text { Intercouplet } \\
\text { distance }(\mathrm{cm})\end{array}$ & $\begin{array}{l}\text { Overtrack } \\
(\mathrm{cm})\end{array}$ & $\begin{array}{l}\text { Avg. interior } \\
\text { straddle }(\mathrm{cm})\end{array}$ & $\begin{array}{l}\text { L/R Lateral offset } \\
(\text { Cross measure })(\mathrm{cm})\end{array}$ \\
\hline $15 \mathrm{a}$ & ca. 4.5 & 256.5 & $81.3,81.3$ & $-26.7,-26.7$ & -2.5 & $-5.1(27.3),-5.1(27.3)$ \\
$15 \mathrm{~b}$ & 6.14 & 308.6 & $108.0,111.8$ & $-27.9,-27.9$ & -6.6 & $-5.1(28.3),-5.1(28.3)$ \\
& & & & & & \\
$20^{\mathrm{V}}$ & ca. 4.04 & ca. 223.0 & ca. $60.0,70.0$ & ca. $-23.0,-30.0$ & ca. -1.1 & ca. $-6.9(23.0), 6.9(30.5)$ \\
$16^{\mathrm{V}}$ & ca. 4.41 & ca. 252.0 & ca. $77.0,77.0$ & ca. $-35.0,-35.0$ & ca. -2.5 & ca. $-8.7(36.1), 8.7(36.1)$ \\
$17^{\mathrm{V}}$ & ca. 5.43 & ca. 302.4 & ca. $104.1,104.1$ & ca. $-33.1,-33.1$ & ca. -2.5 & ca. $-8.7(34.2), 8.7(34.2)$ \\
& & & & & & \\
$22 \mathrm{a}^{\mathrm{K}}$ & ca. 2.13 & ca. 150.0 & ca. $45.0,37.5$ & ca. $-17.5,-17.5$ & ca. -3.3 & ca. $-2.0(17.6), 3.0(17.7)$ \\
$22 \mathrm{~b}^{\mathrm{K}}$ & ca. 2.69 & ca. 172.5 & ca. $37.5,30.0$ & ca. $-30.6,-36.3$ & ca. -0.8 & ca. $-4.0(30.9), 2.0(36.4)$ \\
\hline
\end{tabular}

Table S8: Measurements of the pace

\begin{tabular}{|lllllll|} 
Horse & $\begin{array}{l}\text { Calculated } \\
\text { speed }(\mathrm{m} / \mathrm{s})\end{array}$ & $\begin{array}{l}\text { Cycle } \\
\text { length }(\mathrm{cm})\end{array}$ & $\begin{array}{l}\text { Intercouplet } \\
\text { distance }(\mathrm{cm})\end{array}$ & $\begin{array}{l}\text { Overtrack } \\
(\mathrm{cm})\end{array}$ & $\begin{array}{l}\text { Avg. interior } \\
\text { straddle }(\mathrm{cm})\end{array}$ & $\begin{array}{l}\text { L/R Lateral offset } \\
\text { (Cross measure })(\mathrm{cm})\end{array}$ \\
\hline $18^{\mathrm{V}}$ & ca. 3.11 & ca. 198.0 & ca. $58.4,58.4$ & ca. $27.9,27.9$ & ca. 0 & ca. $-10.2(27.9), 10.2(27.9)$ \\
$19^{\mathrm{V}}$ & ca. 7.92 & ca. 371.2 & ca. $140.0,140.0$ & ca. $15.2,15.2$ & ca. 0 & ca. $-12.2(27.4), 12.2(27.4)$ \\
$23^{\mathrm{K}}$ & ca. 8.80 & ca. 352.9 & ca. $94.0,117.5$ & ca. $25.9,17.6$ & ca. -5.1 & ca. $-5.1(26.1), 5.1(18.3)$ \\
\hline
\end{tabular}


Table S9: Measurements of the medium canter. There is only a single couplet so lateral offset also indicates whether directionality is to left $(\mathrm{L})$ or right $(\mathrm{R})$

\begin{tabular}{|lllllll|} 
Horse & $\begin{array}{l}\text { Calculated } \\
\text { speed } \\
(\mathrm{m} / \mathrm{s})\end{array}$ & $\begin{array}{l}\text { Cycle } \\
\text { length } \\
(\mathrm{cm})\end{array}$ & $\begin{array}{l}\text { Distance between } \\
\text { steps }\end{array}$ & $\begin{array}{l}\text { Overtrac } \\
\mathrm{k}(\mathrm{cm})\end{array}$ & $\begin{array}{l}\text { Interior } \\
\text { straddle } \\
1-1(\mathrm{~cm})\end{array}$ & $\begin{array}{l}\text { L/R Lateral } \\
\text { offset } \\
\text { (Cross measure) } \\
(\mathrm{cm})\end{array}$ \\
\hline 14 & 3.72 & 227.6 & $42.3,69.9,45.7$ & 8.9 & 0 & $-2.5 \mathrm{~L}(24.3)$ \\
9 & 3.86 & 241.4 & $63.5,66.0,50.8$ & 5.1 & 5.1 & $-6.4 \mathrm{~L}(20.0)$ \\
2 & 4.06 & 240.0 & $38.1,106.7,45.7$ & -5.1 & 0 & $-1.3 \mathrm{R}(11.6)$ \\
5 & 4.12 & 241.0 & $58.4,73.6,53.3$ & -5.1 & 1.9 & $1.9 \mathrm{~L}(10.3)$ \\
13 & 4.14 & 251.6 & $38.1,86.4,71.1$ & 0 & 5.1 & $-10.5 \mathrm{~L}(15.1)$ \\
1 & 4.49 & 264.2 & $63.5,90.2,61.0$ & -1.3 & 2.5 & $-6.4 \mathrm{R}(13.1)$ \\
6 & 4.80 & 264.0 & $63.5,71.1,63.5$ & 5.1 & 0 & $-11.4 \mathrm{~L}(23.0)$ \\
10 & 5.35 & 287.1 & $81.3,55.9,85.1$ & 14.0 & 0 & $6.4 \mathrm{~L}(27.3)$ \\
& & & & & & \\
$24^{\mathrm{K}}$ & 3.28 & ca. 195.0 & ca. $41.3,48.8,33.8$ & ca. 13.1 & ca. 0 & ca. $-6.7 \mathrm{R}(26.4)$ \\
$21^{\mathrm{K}}$ & 5.80 & ca. 315.0 & ca. $90.0,108.8,67.5$ & ca. -12.7 & ca. 5.1 & ca. 5.1L $(5.1)$ \\
\hline
\end{tabular}

Table S10: Measurements of the gallop

\begin{tabular}{|lllll|} 
Horse & $\begin{array}{l}\text { Calculated } \\
\text { speed } \\
(\mathrm{m} / \mathrm{s})\end{array}$ & $\begin{array}{l}\text { Cycle } \\
\text { length } \\
(\mathrm{cm})\end{array}$ & $\begin{array}{l}\text { Distance between steps } \\
(\mathrm{cm})\end{array}$ & $\begin{array}{l}\text { Avg. interior } \\
\text { straddle }(\mathrm{cm})\end{array}$ \\
\hline 8 & 4.78 & 274.2 & $27.9,68.6,40.6,71.1$ & 5.1 \\
11 & 5.43 & 296.0 & $36.8,58.4,71.1,73.7$ & 15.2 \\
5 & 5.52 & 286.9 & $12.7,50.8,101.6,61.0$ & 10.2 \\
1 & 6.48 & 329.0 & $39.4,67.3,97.8,73.7$ & 5.1 \\
9 & 7.11 & 348.1 & $25.4,66.0,124.5,76.2$ & 10.2 \\
& & & & \\
$25^{\mathrm{K}}$ & ca. 4.92 & ca. 262.5 & ca. $41.3,56.3,52.5,48.8$ & ca. -3.0 \\
\hline
\end{tabular}

90 Figure S1. Illustrations of irregularities in foot imprints in the walk and trot of modern horses

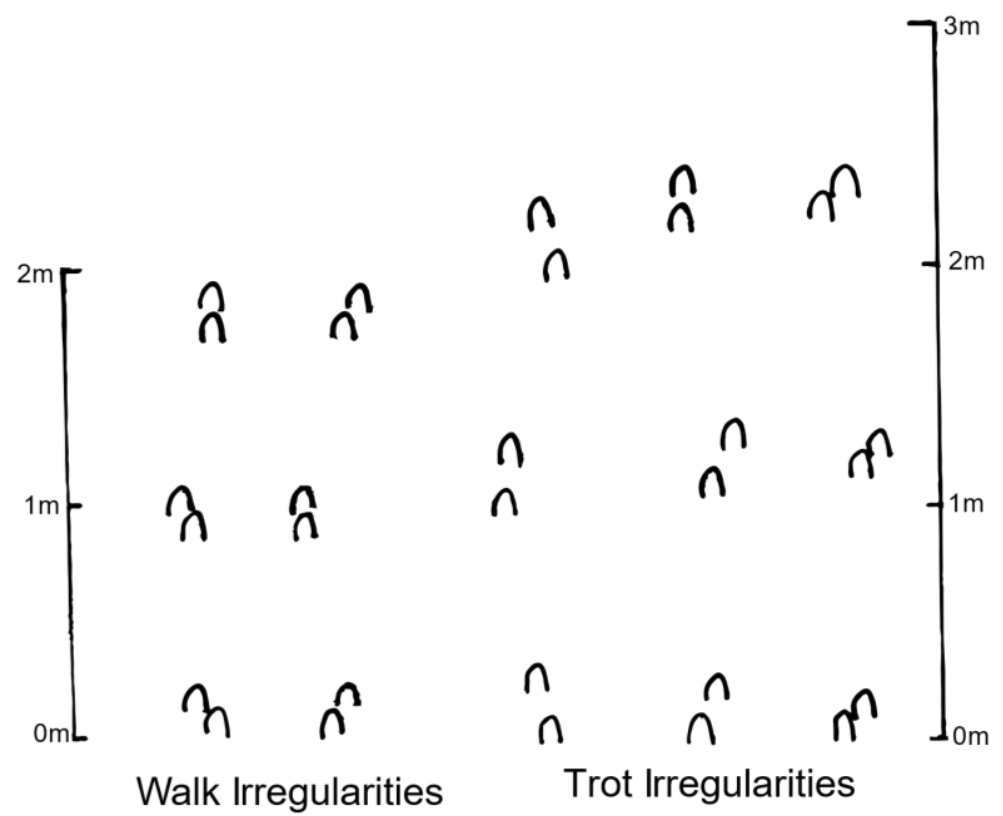


Figure S2. Photographs of Irregular Gaits

95
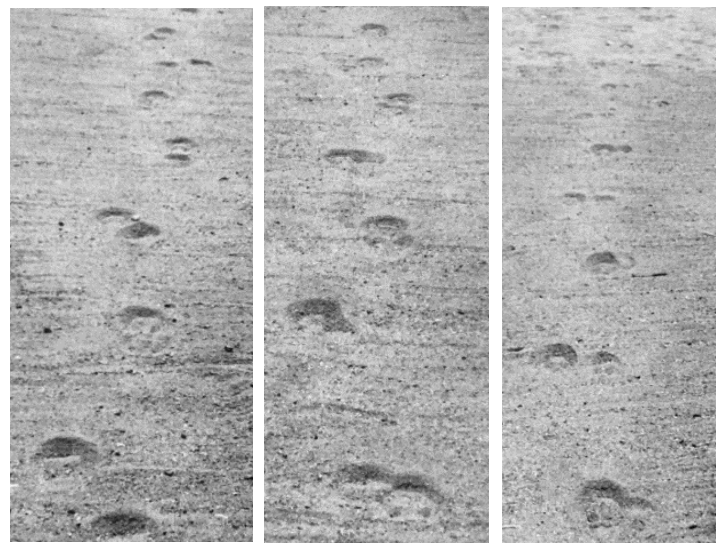

a. Irregular Walks

(Horses 2 (fast), 2 (slow), and 14 (slow))
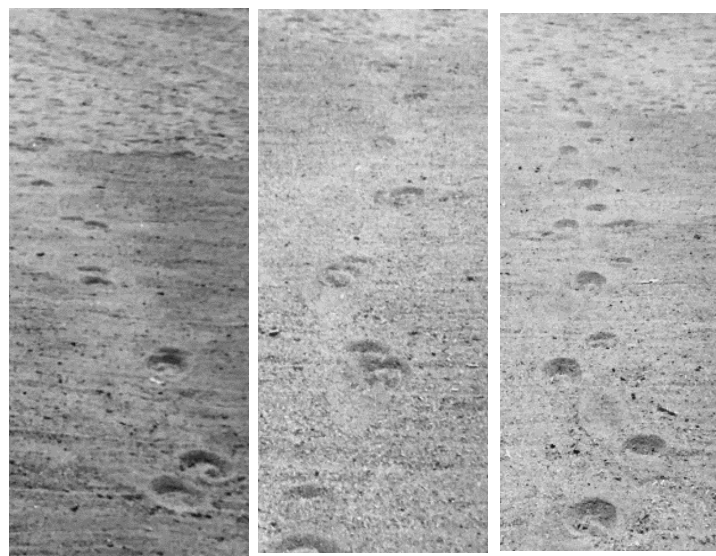

b. Irregular Trots

(Horses 2 (fast), 3 (fast), 14, (slow)) 